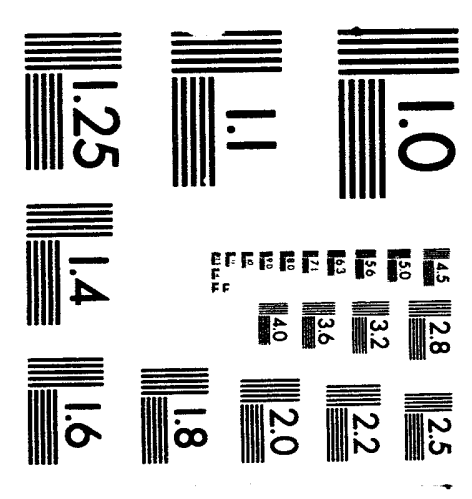



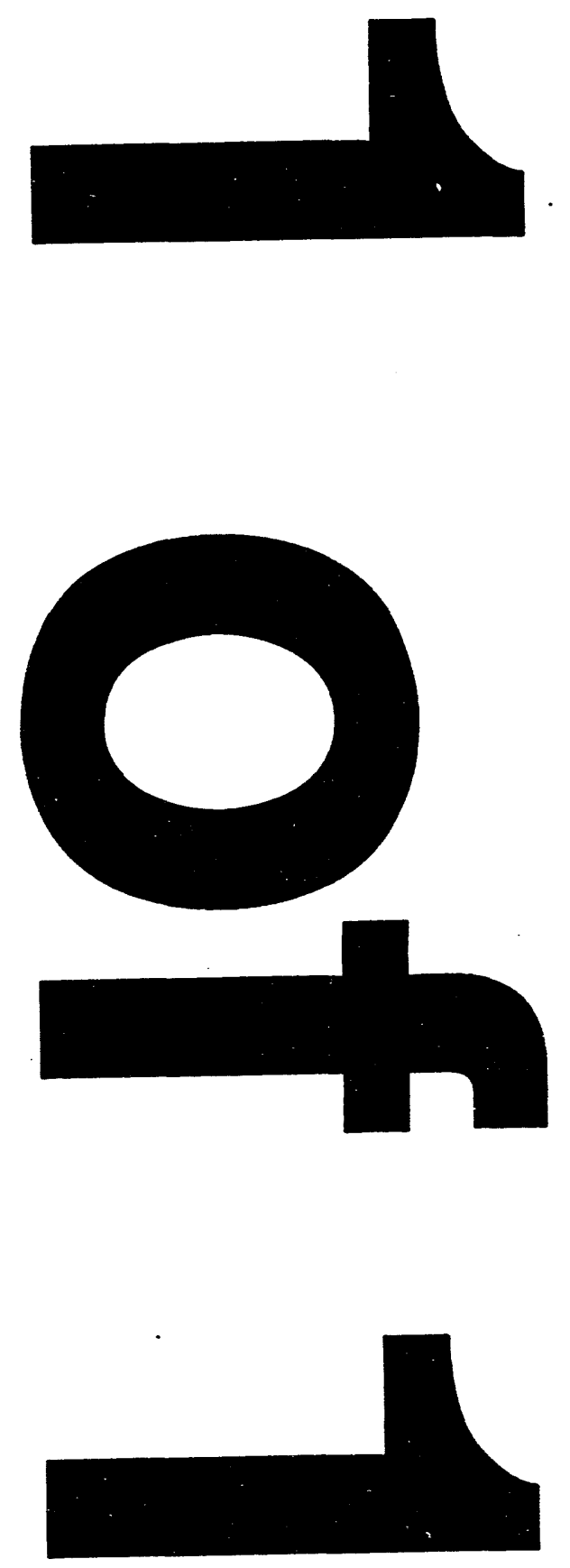


\title{
LIGHT SCATTERING INVESTIGATION OF PHASE SEPARATION IN A MICELLE SYSTEM
}

\author{
Jess P. Wilcoxon, James E. Martin and Judy Odinek \\ Advanced Materials Physics Division \\ Sandia National Laboratories, Albuquerque, New Mexico 87185
}

\section{ABSTRACT}

We report a real-time, two-dimensional light scattering study of the evolution of structure in a two component nonionic micelle system during phase separation via spinodal decomposition. Our principal finding is that domain growth proceeds much slower than the cube root of time prediction for simple binary fluids. In fact, the growth kinetics can be empirically described as a stretched exponential approach to a pinned domain size. Although the kinetics are not yet understood, this anomalous behavior may be due to the ability of the spherical micelles to reorganize into more complex structures. The domain structure also shows some anomalies. Although at short times the expected structure factor for a critical quench is observed, at long times the structure factor crosses over to the off-critical form. However, in all cases the average scattered intensity is proportional to the cube of the domain size. These findings are discussed in comparison to standard theories of and experimental work on binary fluids.

\section{DISCLAIMER}

This report was prepared as an account of work sponsored by an agency of the United States Government. Neither the United States Government nor any agency thereof, nor any of their employees, makes any warranty, express or implied, or assumes any legal liability or responsibility for the accuracy, completeness, or usefulness of any information, apparatus, product, or process disclosed, or represents that its use would not infringe privately owned rights. Reference herein to any specific commercial product, process, or service by trade name, trademark, manufacturer, or otherwise does not necessarily constitute or imply its endorsement, recommendation, or favoring by the United States Government or any agency thereof. The views and opinions of authors expressed herein do not necessarily state or reflect those of the United States Government or any agency thereof. 


\section{INTRODUCTION}

Micelles are surfactant aggregates with a droplet-like structure that can form in either polar or nonpolar solvents. In nonpolar solvents such as oils the hydrophobic (lyophilic) surfactant tails are exposed to form reverse or inverse micelles. Micelles formed of nonionic surfactants often phase separate when the temperature is increased or decreased (inverse systems). The critical point behavior of these systems has been the subject of several investigations[1-5] and it has been established[2-5] that they fall into the same universality class as simple binary fluids, although the critical concentration is quite low due to the large size of the micelles compared to the solvent. In particular, static properties, such as the divergence of the correlation length and the osmotic compressibility, can be expressed as power-laws in the reduced temperature with Ising exponents.

Dynamical scaling has also been observed in micellar solutions. Critical slowing down occurs near the critical point and the decay of the intensity autocorrelation function in the single phase region is exponential, in accord with the Kawasaki mode-mode coupling theory. In fact, the entire static and dynamic critical behavior of micelle solutions is indistinguishable from that of simpie binary fluids, although the length scale amplitude is roughly an order of magnitude larger.

However, the universal Ising-type critical behavior of these systems does not extend deeply into the two phase region. Several years ago we observed [6] that a sample equilibrated in the twophase region exhibits unusually strong scattering due to structures of enormous size. This novel scattering occurs in both inverse and normal micelles. It is reasonable to expect the formation of large structures to affect the kinetics of phase separation. In this paper we report the first study of the evolution of structure in a micelle system in an attempt to understand the origin of these structures.

We first present measurements of the structure factor for a shallow temperature quench that show that there is no measureable regime, at least by our methods, that can be described by the linear Cahn-Hilliard theory.[7] In this linear theory it is expected that the ring intensity, or domain mass, grows exponentially while the characteristic length scale, or domain size, is constant. Instead, we find that the domain size increases as the cube root of time - the standard Lifshitz- 
Slyozov [8] result - for the shallowest quenches. Furthermore, at all quench depths the relation between the domain mass and size indicate the development of non-fractal domains, as expected for spinodal growth. We then demonstrate that the structure factor has a form that is well described by the Furukawa function, albeit with a significant deviation at large scattering wavevectors, and a crossover from critical to off-critical behavior at long times.

As the quench depth increases, the growth kinetics deviates progressively from a power law in time, becoming slower than any power law. In fact, all of the data can be described by an asymptotic approach of the domain size to a value of about $25 \mu \mathrm{m}$. This approach is well described by a stretched exponential of time with an exponent of $1 / 2$. We speculate that the ultraslow kinetics observed for deep quenches might be due to the formation of the large structures we have previously observed in the "equilibrium," fully separated two phase system.

\section{EXPERIMENTAL}

\section{Sample Preparation}

Previous work [6] has demonstrated that nonionic surfactants are sensitive to oxygen, so all samples were prepared in a Vacuum Atmospheres Argon-filled glove box. The nonionic surfactant used in this study was n-dodecylhexaoxyethylene glycol monoether which we abbreviate hereafter as $\mathrm{C}_{12} \mathrm{E}_{6}$. In previous work [6] we showed how addition of a simple salt, $\mathrm{NaCl}$, could be used to adjust the critical temperature without affecting the static or dynamic scaling behavior. In this case $10 \mathrm{wt} \% \mathrm{NaCl}$ in filtered HPLC grade water was added to pure $\mathrm{C}_{12} \mathrm{E}_{6}$ to adjust the phase transition temperature to $\mathrm{T}_{\mathrm{C}} \cong 30.70{ }^{\circ} \mathrm{C}$ at a critical concentration of $\mathrm{c}_{\mathrm{C}} \equiv 2 \mathrm{wt} \% \mathrm{C}_{12} \mathrm{E}_{6}$ (the actual critical temperature was determined daily as it tended to increase with time). The sample was sealed in a cylindrically shaped cell with a $1 \mathrm{~mm}$ path length. This short path length minimizes multiple scattering.

We chose to work with a water continuous normal micelle system since the density of water and the nonionic surfactant is nearly identical. This minimizes convection during phase separation, thus allowing us to follow the spinodal decomposition for a long time. The phase diagram of our 
system was reported previously. [6] As expected, it is quite asymmetrical, due to the size difference between the micelles and the water.

\section{Temperature Quenches}

Temperature is the key control parameter in these experiments. For good temperature stability a large thermal mass is required, but we must also be able to rapidly quench the sample. We used a commercial 10 gallon aquarium (Walmart ${ }^{\mathrm{TM}}$ stores) and supported our $2 \mathrm{ml}$ sample cell in the aquarium by a rod mounted on a precise translational positioning stage. The temperature was monitored with an Hewlett-PackhardP ${ }^{\mathrm{TM}}$ quartz thermometer whose resolution is either $1 \mathrm{mK}$ (1 second sampling) or $0.1 \mathrm{mK}$ (10 sec sampling). A Tronac ${ }^{\mathrm{TM}}$ controller allowed temperature quenches as small as $2 \mathrm{mK}$ while controlling the temperature to $\pm 0.2 \mathrm{mK}$ under ideal circumstances. A powerful stirrer ensured rapid heat distribution throughout the bath and the heater and stirrer were positioned to optimize bath performance. Temperature quenches were effected by injecting a calibrated amount of $50^{\circ} \mathrm{C}$ water to the bath which was initially $\sim 10 \mathrm{mK}$ below $\mathrm{T}_{\mathrm{c}}$ (recall that this system exhibits a LCST).

The sample cell had a $1 \mathrm{~mm}$ path length to allow for rapid temperature equilibration. In shallow quenches the bath/sample temperature equilibrated before the appearance of the spinodal ring. However, for quenches of greater than $\sim 50 \mathrm{mK}$, a ring appeared prior to temperature equilibration, due to the more rapid onset of spinodal decomposition for deep quenches. This limited us to quenches shallower than $50 \mathrm{mK}$.

\section{Two-dimensional light scattering}

To study the kinetics of phase separation requires the ability to determine structure as a function of time. Traditional one-dimensional light scattering instruments must repetitively scan through a sequence of angles, with the result that data are acquired in an interval during which the structure is evolving, so that the temporal resolution is compromised. We have developed [20] a two-dimensional, time-resolved light scattering instrument that is based on currently available video and computer technology. 
A laser beam, focused with a $40 \mathrm{~cm}$ focal length lens, illuminates the sample, the scattered light impinges on a diffusing screen, and is collected by a fixed-gain Pulnix ${ }^{\mathrm{TM}}$ video camera and stored on a VCR at a spatial resolution of $640 \times 480$. The video frames are then grabbed from the tape and 8 bit digitized by a Perceptics PixelBuffer ${ }^{\mathrm{TM}}$ card on a Macintosh Quadra $950^{\mathrm{TM}}$. The frame grabber card immediately transfers these images to a 32 Mbyte dual-ported PixelStore ${ }^{\mathrm{TM}}$ memory board that resides on both the slow computer bus and a very fast direct bus from the frame grabber that can support the data transfer rate of $10 \mathrm{Mbyte} / \mathrm{sec}$. The digitized images have a dynamic range of 256 , a spatial resolution of $512 \times 480$, and a temporal resolution of $1 / 30 \mathrm{sec}$. Using $454.5 \mathrm{~nm}$ light, the length scale regime that can be studied is from $2 \pi / \mathrm{q}=22 \mu \mathrm{m}$ to $0.95 \mu \mathrm{m}$, where $q=4 \pi \sin (\theta / 2) / \lambda$ is the scattering wave vector, $\theta$ is the scattering angle and $\lambda$ is the wavelength in the scattering medium. The intensity is analytically corrected for the incident polarization of the laser, polarization and incident angle dependent cell, bath and screen reflectances, the emerging angular distribution of light from the diffusing screen, camera vignetting, the Jacobian for light refraction and the Jacobian of the projection of a sphere onto a flat screen (twice). Calibration runs with a uniform scatterer yielded a constant image after these corrections were applied. The video tape was started just prior to initiating the temperature quench which allowed us to collect a background frame for subtraction from each time-resolved quench frame. The success of this method was evidenced by the completely flat scattering curve for the $t=0$ frame.

Finally, radial averaging the scattering data allows high quality data to be obtained despite the limited dynamic range (0-255) of the video digitizer. Since roughly $10^{3}$ pixels are averaged for each intensity bin, the dynamic range is increased by a factor of about 30 .

\section{STRUCTURE}

\section{Shallow Quenches}

Radially-averaged scattering data taken during a $9 \mathrm{mK}$ quench - we refer to such shallow quenches as "quenchettes" - are shown in Fig. 1 (although our time resolution is 33 ms we have only shown a few data sets for clarity). An unstable concentration fluctuation that intensifies with 
time and moves toward $q=0$ is clearly observed. A significant feature of the data is the pronounced maximum, or spinodal ring, that occurs at a scattering wavevector $\mathrm{q}_{\max }$ and has an intensity $I_{\max }$. The peak position and amplitude have simple physical interpretations: the characteristic domain size is just $L(t)=2 \pi / q_{\max }$ and the characteristic domain mass- i.e. the domain mass within a domain size, is just proportional to $I_{\max }$. Key issues are the structure of the domains and their growth kinetics.

A salient feature of these data is that as soon a ring can be resolved its peak position is already moving toward $q=0$, in contradiction to the linear Cahn-Hilliard [7] theory, which predicts exponential growth of a stationary ring at early times. In real space, the Cahn-Hilliard description corresponds to phase separation into domains of fixed size but of increasing concentration difference. Despite our best efforts to find this linear regime we were unsuccessful. Of course, one can always postulate that the linear regime occurs on time scales shorter than can be resolved, so although we can't rule out Cahn-Hilliard, we can't measure it either.

Having resolved that the domains grow from the earliest observable times, an obvious issue is whether the domain structures grow by simple enlargement, while their morphology is statistically constant. If this is case then the scattering data will collapse onto a master curve when plotted on the universal axes $I / I_{\max }$ versus $q / q_{\max }$. In fact, a good data collapse, shown in Fig. 2 , is obtained, indicating that the expected scaling of the structure does occur, at least for shallow quenches. However, the form of the collapsed data differs in some regards from standard results, as we shall now discuss.

For spinodal decomposition in simple liquids (as well as other systems) Furukawa [10] has presented heuristic arguments that the scattered intensity $I(q, t)$ has the universal scaling form:

$$
I(q, t) \sim q_{\max }^{-3} f(x)
$$

where $x=q / q_{\max }$ and $q_{\max }$ is a function of time. The scaling function depends on whether the quench is along the critical isochore and has the form 


$$
\begin{aligned}
f(x) & =\frac{4 x^{2}}{3+x^{8}} ; \quad \text { critical quench } \\
& =\frac{3 x^{2}}{2+x^{6}} ; \quad \text { off }- \text { critical quench }
\end{aligned}
$$

These functions have simple limiting behaviors at small and large wavevectors,

$$
\begin{aligned}
& f(x) \sim x^{2} \quad x<1 \\
& \sim x^{-6} \quad x \gg>1 \text {; critical quench } \\
& \sim x^{-4} \quad x>>1 \text {; off }- \text { critical quench }
\end{aligned}
$$

that can easily be discerned in the data. At small $q$ the structure factor increases as $q^{2}$, which is simply the result of having a conservation law. For large $\mathrm{q}$ there is a $\mathrm{q}^{-4}$ decrease for an offcritical quench, which is simply Porod's scattering law for sharp interfaces. The $q^{-6}$ fall-off is for critical quenches and is apparently due to a more complex interface.

To what extent is this expected scaling evident in our critical quench data? The data do increase as $\mathrm{q}^{2}$ for $\mathrm{q}<\mathrm{q} \max$ and fall-off as $\mathrm{q}^{-6}$ for $\mathrm{q}>\mathrm{qmax}$, agreement with Eq. 3. However, at higher values the $\mathrm{q}^{-6}$ fall-off crosses over to $\mathrm{q}^{-2}$, possibly indicating the formation of more complex structures, such as micellar aggregates. Or perhaps this is due to the fact that a system near the critical point has a subtantial correlation length beneath which Ornstein-Zernike scattering can be observed. In other words, critical opalescence occurs within the domain structures once the domain size is greater than the correlation length.

In any case, it is clear that the theory of phase separation of binary solutions does not normally admit the possibility that one component, in this case the spherical micelles, can reorganize into more complex structures. The scaled data for the $2 \mathrm{mK}$ quench show the same features found for the $9 \mathrm{mK}$ quench: $\mathrm{a} \mathrm{q}^{-6}$ to $\mathrm{q}^{-2}$ crossover occurs. Thus although scaling of the domain structures does occur, an unexpected feature appears at high $q$ that we do not fully understand. 
The next issue is how the domain size depends on the domain mass. For non-mass fractal objects in three dimensions it is expected that the mass increases as the cube of a length. Since the peak intensity is a domain mass and the inverse of the peak position is the domain size we expect to observe

$$
I_{\max }(t) \sim q_{\max }^{-3}(t)
$$

Observation of this relation would imply that the coarsening of non-mass fractal domains is taking place during the phase separation process, independent of quench depth.

To reduce the noise in the characteristic length $L=2 \pi / q_{\max }$ we used the moments

$$
I_{k}\left(q_{1}, q_{u}\right)=\int_{q_{1}}^{q_{u}} q^{k} I(q) d q
$$

In practice, the lower integration limit $\mathrm{q}_{\mathrm{l}}=0.33 \times 10^{-3} \mathrm{~nm}^{-1}$ is determined by the beam stop size and the upper limit $\mathrm{q}_{\mathrm{u}}=0.529 \times 10^{-2} \mathrm{~nm}^{-1}$ is set by the camera position. If the scattered intensity scales according to eq. 1 then the moments that depend only on powers of $\mathrm{q}_{\max }(\mathrm{t})$ can be obtained by scaling so that $q_{l} \sim q_{u} \sim q_{\max }(t)$ : i.e., $q_{\max }(t) \sim I_{1}\left(a q_{\max }, b q_{\max }\right) / I_{0}\left(a q_{\max }, b q_{\max }\right)$, where $a$ and $b$ are arbitrarily chosen to maximally exploit the available data and $q_{\max }(t)$ is found iteratively. These partial scaled moments are strictly proportional to the true moments, but in practice we found that simply integrating over the finite data gave moments that scaled similarly to the partial scaled moments.

The final result, shown in Fig. 3, follows Eq. 3 closely, especially given the deviations observed in the structure factor itself. Evidently, this integrated measure of structure is much less sensitive to morphology.

Scattering data for deeper quenches were quantitatively similar to the shallow quench data, albeit a crossover from critical quench to off-critical quench behavior was seen at large times. In 
particular, the same cubic dependence of the domain size on the domain mass was observed, and the structure factor was found to scale on dimensionless axes, with $q^{2}, q^{-6}$ and $q^{-2}$ regimes. However, as we shall now discuss, the kinetic data show large differences between the shallow and deep quench data.

\section{KINETICS}

It is of great interest to determine whether the structural anomalies we have observed are manifest in the kinetics of structure formation. For a two-component system with a conserved order parameter the well known prediction [8] of Lifshitz-Slyozov is that the domain size should increase as the cube root of time, $\mathrm{L} \sim \mathrm{t}^{1 / 3}$, and in fact we do observe this scaling for the shallowest quenches, as shown in Fig. 4. However, as the quench depth increases we see progressively larger deviations from Lifshitz-Slyozov, with the kinetics becoming much slower than expected. (This is contrary to the linear late time hydrodynamic coarsening of Siggia [11].) And of course, since $\mathrm{I}_{\mathrm{m}} \sim \mathrm{L}_{\mathrm{m}}{ }^{3}$ for all quench depths investigated, the slow kinetics is manifest in the intensity.

This finding led to a flurry of nonlinear curve fitting on our part, in an attempt to determine the growth law. Our first concrete finding is that a power law description of the growth kinetics fails utterly as the quench depth increases; in fact, the nonlinear fit attempts to set the exponent to zero. Sounds logarithmic, right? Well a plot against log time looks OK in most cases, but again the data tend to deviate for the deeper quenches. On the basis of a simulation of random impurities, we then fit the data to $L(t)=L(0)+a[\ln (t)]^{X}$ and found that the exponent $x$ varied systematically with quench depth, and that the 3-parameter fits were often not all that good, all of which was not very satisfying.

As shown in Fig. 5 the growth exponent changes with quench depth, the smallest exponent of 0.9 is required to fit the data for the deepest quench $(37 \mathrm{mK})$ while an exponent of 3.2 can describe the data for the $2 \mathrm{mK}$ quench. However, though the fits shown are excellent over the entire time regime, the requirement to vary the exponent to reflect the slower growth kinetics for deeper quenches is unsatisfactory. 
At the suggestion of S. Glotzer, who works on spinodally decomposing systems that pin, we then attempted to analyze our data in terms of an asymptotic approach to some pinning length, i.e.

$$
L(t)=L(\infty)\left(1-e^{-\left(\frac{t}{\tau}\right)^{\beta}}\right)
$$

As with the power law and logarithmic fits, this function has 3 adjustable parameters, $L(\infty), \tau$, and $\beta$. However, it turned out that the stretched exponential exponent ended up being very close to 0.5 for all quench depths, while the pinning length $L(\infty)$ was nearly constant at a value of $\sim 25 \mu \mathrm{m}$. We really felt we might be on to something now! We thus decided to fix these parameters and let only the characteristic time $\tau$ vary with quench depth. A semi-log plot of the reduced length $(L(\infty)$ $-\mathrm{L}(\mathrm{t})) / \mathrm{L}(\infty)$ versus $\mathrm{t}^{0.5}$ should result in a straight line for each of the quench depths. The plot shown in Fig. 6 demonstrates that this is a good description of all our data except the very deepest quench. An important point, however, is that a functional form that approaches an asymptote can be a misleading fit unless the pinning length is reasonably close to the largest length scale in the experimental data. Our data approaches $80 \%$ of the pinning length, so we do not not think that the pinning interpretation can be rejected out of hand.

\section{DISCUSSION}

The stretched exponential analysis now begs the question of whether the postulated pinning is extrinsic- due perhaps to impurities- or intrinsic, due to the ability of the spherical micelles to restructure into more complex morphologies. We do not expect impurities of a chemical nature in our samples, and furthermore, any impurities would probably be quickly encapsulated by surfactants and thus rendered benign in their ability to moderate interactions between micelles: after all, this is soap we're talking about here. We suspect that the flattened portion of the phase diagrarn near the critical point leads to phases with large concentration differences even for relatively small quenches. 
In the introduction we motivated these kinetics studies by alluding to previous scattering measurements that showed large structures in the two phase region after phase separation. These measurements were made on the same micellar system herein reported on, but 24 hours after a deep quench. The system then had a well defined meniscus separating a lower, surfactant-poor phase from an upper, surfactant-rich phase. Light and neutron scattering measurements on the surfactant-poor phase, shown in Fig. 7, reveal interfaces of large dimensions, although one might expect to observe only small spherical micelles! In fact, the Porod's law scattering on short length scales is consistent with small micelles, but these apparently organize into larger assemblies. The long-wavelength Porod regime which resembles the asymptotic scaling of the Furukawa dynamic structure factor is quite unexpected and may indicate that the system is still approaching equilibrium. The physical origin of this phenomena remains a mystery, but the common observation $[12,13]$ of such large, apparently non-equilibrium structures in a wide range of surfactant systems indicates that the surface active nature of one of the chemical components is a key factor.

In any case, it seems that the universal critical point behavior of normal and inverse micelles [14] in the single phase region does not extend to the evolution of structure during phase separation. In fact, the kinetics of phase separation is much slower than that reported for either binary fluids [12] or metals [13]. However, a review of the literature indicates that even these simple model systems are not well understood. Wong and Knobler [15] have reported that for a binary fluid the phase separation kinetics could only be described as a power law if the exponent is permitted to depend on quench depth. Furthermore, the exponent used in their analysis had limiting values of 0.3 and 1.1 for intermediate and long times respectively, presumably due to a crossover to hydrodynamic coarsening at long times. In a very recent study of binary fluid phase separation Bailey and Cannell [16] reported that $S(q, t)$ evolved more rapidly than predicted by the best modern theory of Kawasaki and Ohta [17], although dynamic scaling was obeyed. In the case of an $\mathrm{Al}-\mathrm{Zn}$ alloy studied by Hennion et al [18], power-law growth was observed but the exponent 
increased from 0.08 to 0.27 with quench depth. Of course, such small exponents are questionable, and may imply that a power law is not an appropriate description of the kinetics.

We observed that the structure factor has a form that depends somewhat on the domain size. This observation agrees with those of Wong et al. on binary fluids [15] and also with the experiments of Hennion et al. [18] and Katano et al. [19] on metal alloys. In particular, we found that for shallow quenches at early times the intensity grows as $\mathrm{q}^{2}$ for $\mathrm{q}<<\mathrm{qm}_{\mathrm{m}}$ and decays as $\mathrm{q}^{-4}$ for $q \gg q_{m}$, in accord with Furukawa's eq. 1 for off-critical quenches. For deeper quenches or later times a faster $\mathrm{q}^{-6}$ decay was observed. Katano et al. found an $\mathrm{q}^{-4}$ decay at intermediate times that evolved to $\mathrm{q}^{-6}$ for late times in studies of $\mathrm{Fe}-\mathrm{Cr}$ alloys. They noted a concomitant increase from 0.17 to 0.33 in the domain growth exponent. This latter observation is contrary our observation of increasingly slower kinetics (as measured by the slope on a log-log plot) at late times.

\section{CONCLUSION}

The slow kinetics observed in our experiments is not accounte' for by any theory of which we're aware, possibly because of the ability of micelles to reorganize into more complex structures. Admittedly, Lifshitz-Slyozov was observed for the shallowest quench, but the best empirical description for our system appears to be a stretched exponential approach to an asymptote with a fixed exponent of $\beta=0.5$ and fixed pinning value $L(\infty)$ of $\sim 25 \mu \mathrm{m}$. This description is satisfying since only one variable parameter describes virtually all of our data. The anomalous kinetics appears to be related to the large structures observed in previous light and neutron scattering measurements of a phase separated sample in the two-phase region near the critical point.

\section{ACKNOWLEDGEMENT}

We thank the Sharon Glotzer for the suggestion of trying a stretched exponential kinetics description and Jack Douglas for many helpful discussions and his suggestion of $\log (\mathrm{t})$ behavior. This work is supported under DOE contract \#DE-AC04-76DP00789. 


\section{REFERENCES}

1. M. Corti and V. Degiorgio, Phys. Rev. Lett. 55, 2005, (1985).

2. J.P. Wilcoxon and E.W. Kaler, J. Chem. Phys. 86, 4684, (1987).

3. G. Dietler and D.S. Cannell, Phys. Rev. Lett. 60, 1852, (1988).

4. K. Hamano, N Kuwahara, I. Mitsushima, K. Kubota, and T. Kamura, J. Chem. Phys., 94, 2172, (1991).

5. K. Hamano, N Kuwahara, I. Mitsushima, K. Kubota, and J. Ito, Phys. Lett. A 150, 405, (1990).

6. J.P. Wilcoxon, J. Phys. Chem. 94, 7588, (1990).

7. J.W. Cahn and J.E. Hilliard, J. Chem. Phys., 28, 258, (1958).

8. I.M. Lifshitz and V.V. Slyozov, J. Phys. Chem. Solids, 19, 35, (1961).

9. J. E. Martin, J. Odinek, and T. C. Halsey, Phys. Rev. Lett. 69, 1524 (1992).

10. H. Furukawa, Physica, 123A, 497, (1984).

11. Ed Siggia, Phys. Rev. A 20, 595, (1979).

12. D. Roux, J. Physique, 47, 733-738, (1986).

13. J.P. Wilcoxon and E.W. Kaler, J. Chem. Phys. 90, 1909, (1989).

14. J.P. Wilcoxon, Phys Rev A, 43, 1857, (1991).

15. J. Wong and C. Knobler, J. Chem. Phys., 69, 725, (1978).

16. A. Bailey, and D. Cannell, Phys. Rev. Lett., 70, $2110,1993$.

17. K. Kawasaki and T. Ohta , Prog. Theor. Phys., 59, 362, (1978).

18. M. Hennion, D. Ronzaud and P. Guyot, Acta Metall., 30, 599 (1982).

19. S. Katano and M. Iizumi, Phys. Rev. Lett., 52, 835, (1984). 
Figure 1. The growth of the radial-averaged intensity with time, as a function of the momentum transfer q, for a critical quench of $9 \mathrm{mK}$.

Figure 2. When the normalized intensity $\mathrm{I}_{\max }$ is plotted against the normalized momentum transfer $\mathrm{q} / \mathrm{qmax}$ for the $9 \mathrm{mK}$ quench a master curve results that is well described by the Furukawa [10] function for a critical quench. However, at large wavevectors a $\mathrm{q}^{-2}$ tail is observed that is not predicted. This tail may be due to critical opalecscence within the phase separated domains.

Figure 3. The cube of the domain size $\mathrm{L}(\mathrm{t})$ vs the peak intensity $\mathrm{I}_{\max }(\mathrm{t})$ as obtained by moments analysis is plotted for four different quench depths. The best linear fit is shown in each case by the solid line.

Figure 4. The growth of the domain size as a function of time $t$ for four quench depths is plotted against $\mathrm{t}^{1 / 3}$ to determine deviations from Lifshitz-Slyozov growth. Significant deviations in the form of an apparent slowing down occur at large times for deep quenches.

Figure 5. The kinetic data of Fig. 4 are plotted logarithmically. The solid lines through each data set are the best fits to the functional form $L \sim L_{0}+a(\log t)^{x}$. The growth exponent $x$ decreases with increasing quench depth from 3.2 to 0.9 as shown.

Figure 6. The kinetic data of Fig. 4 are fit to a stretched exponential increase to a fixed pinning length. These fits are better than the logarithmic fits, but the physical cause of this apparent pinning behavior is uncertain.

Figure 7. The scattering intensity vs momentum transfer $\mathrm{q}$ for a micelle system quenched deeply into the two phase region and allowed to equilibrate for more than 24 hours. These data were obtained by scattering both light and neutrons (SANS) in the lower, surfactant depleted phase. 


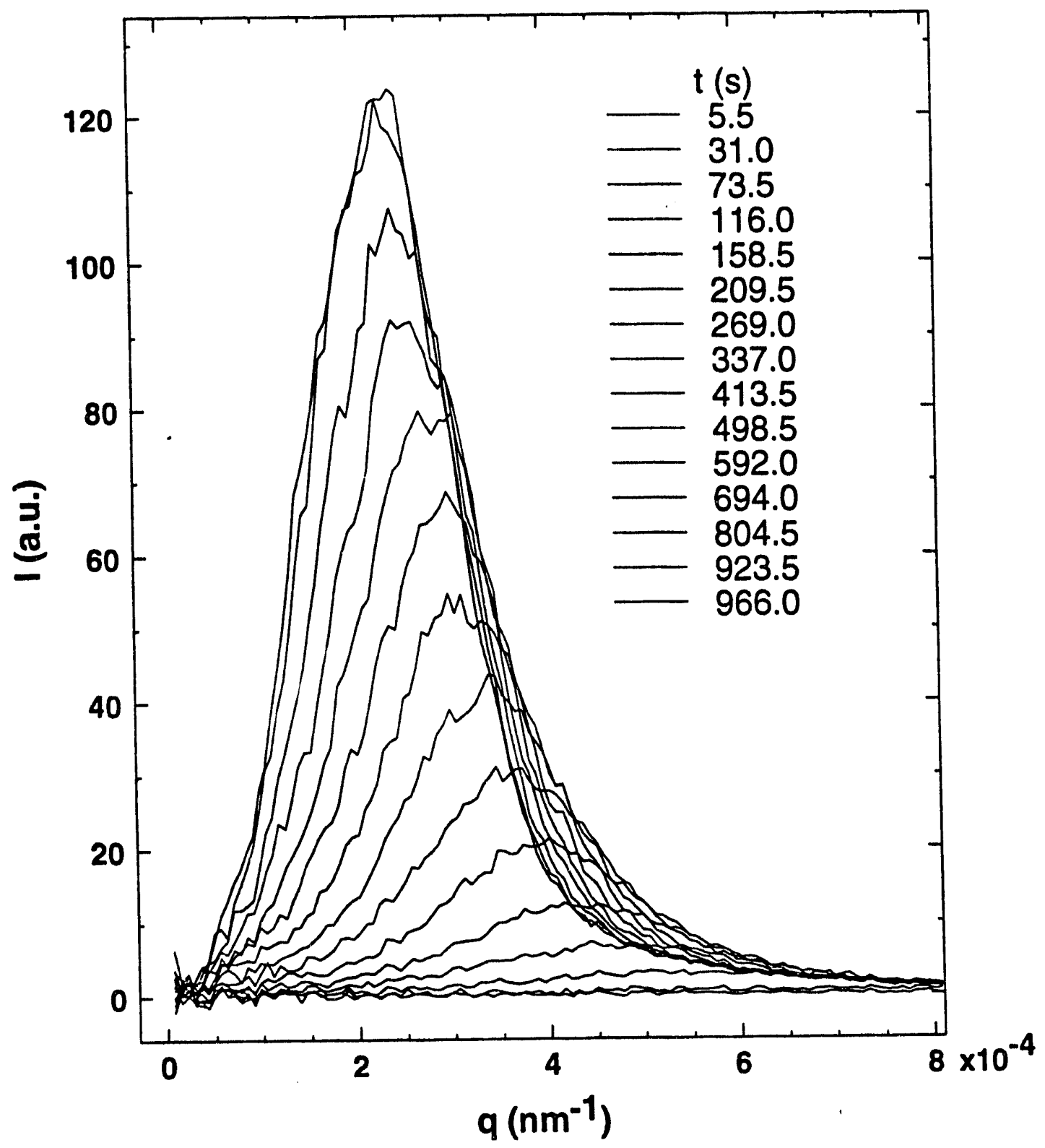




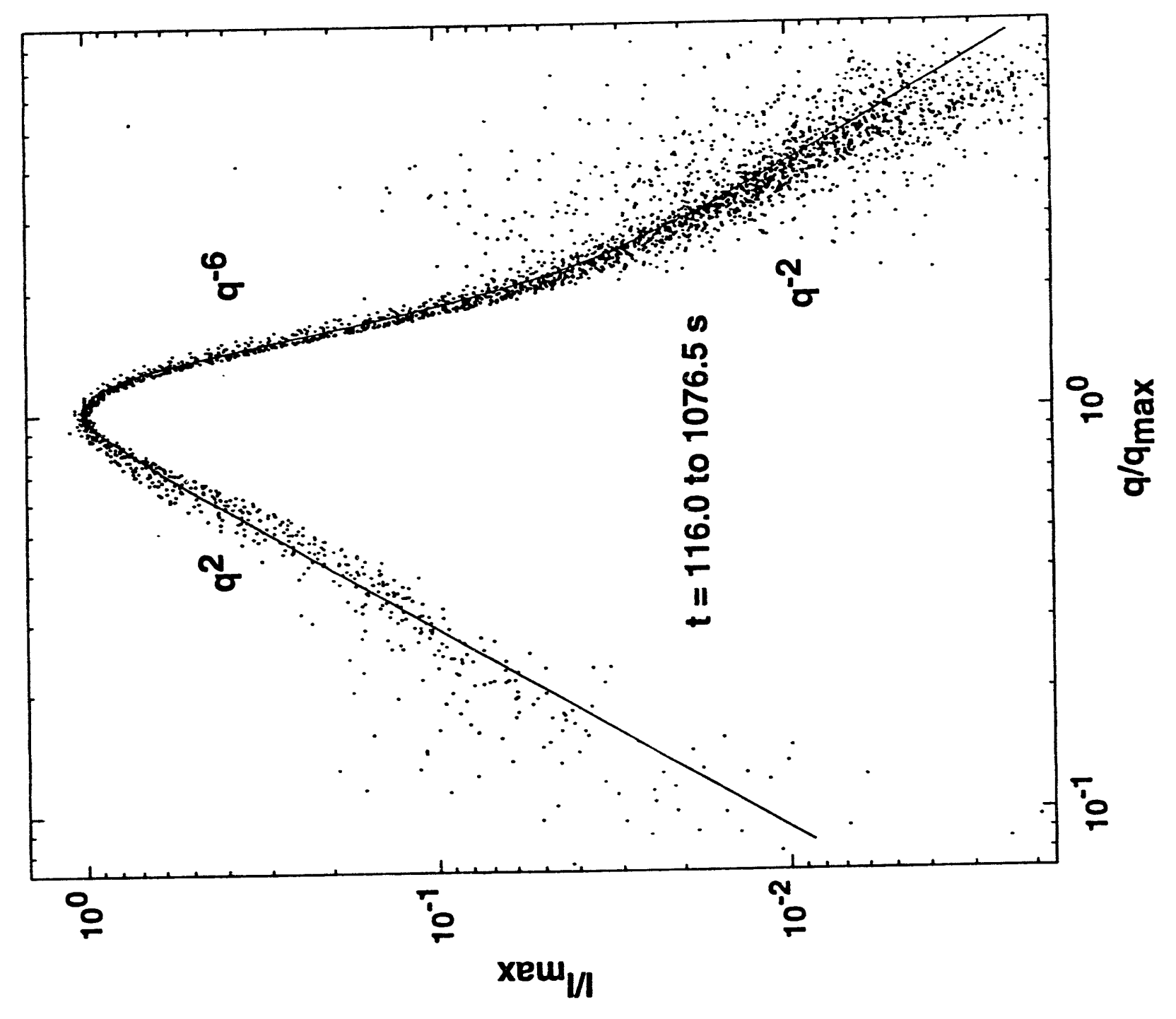




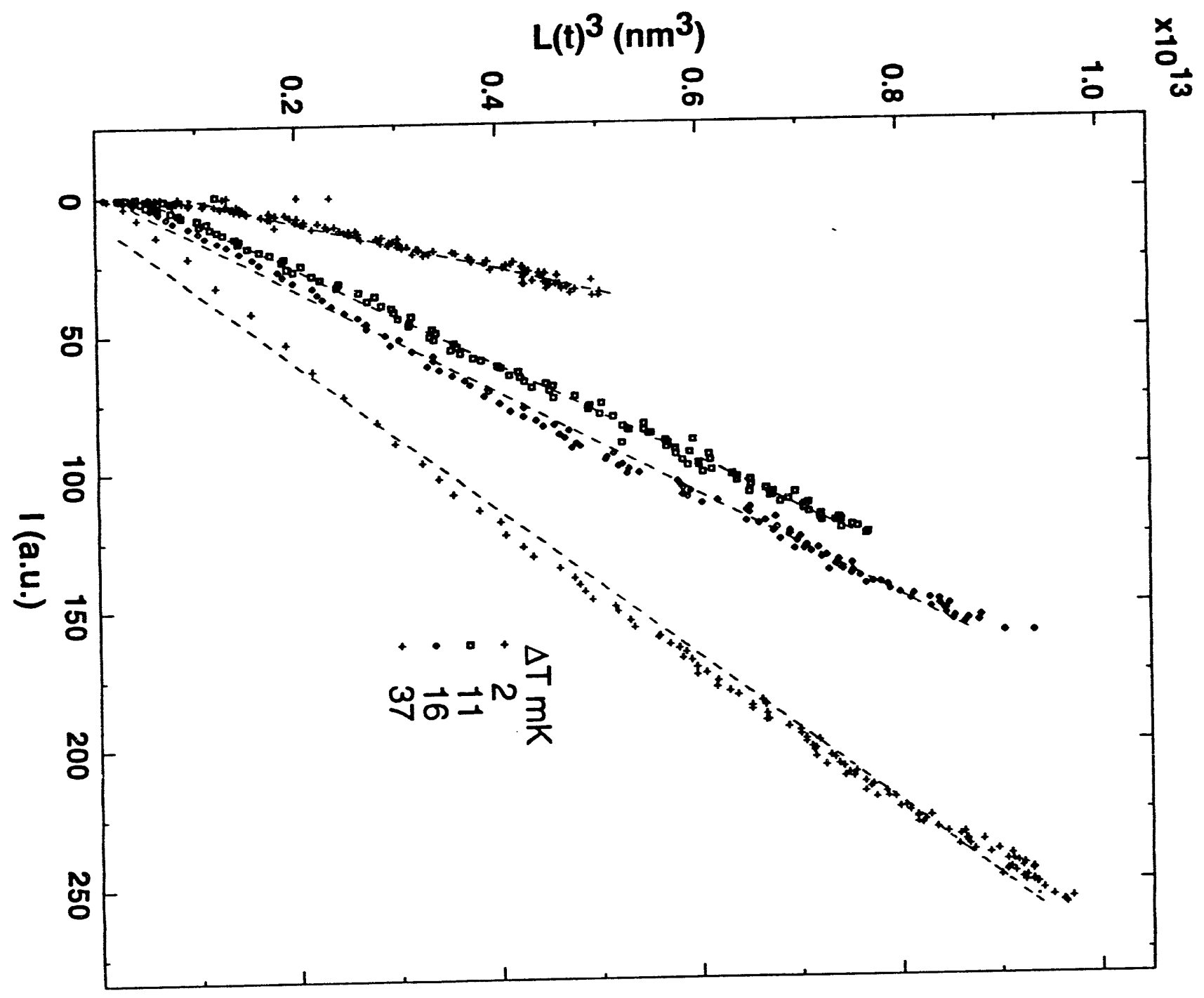




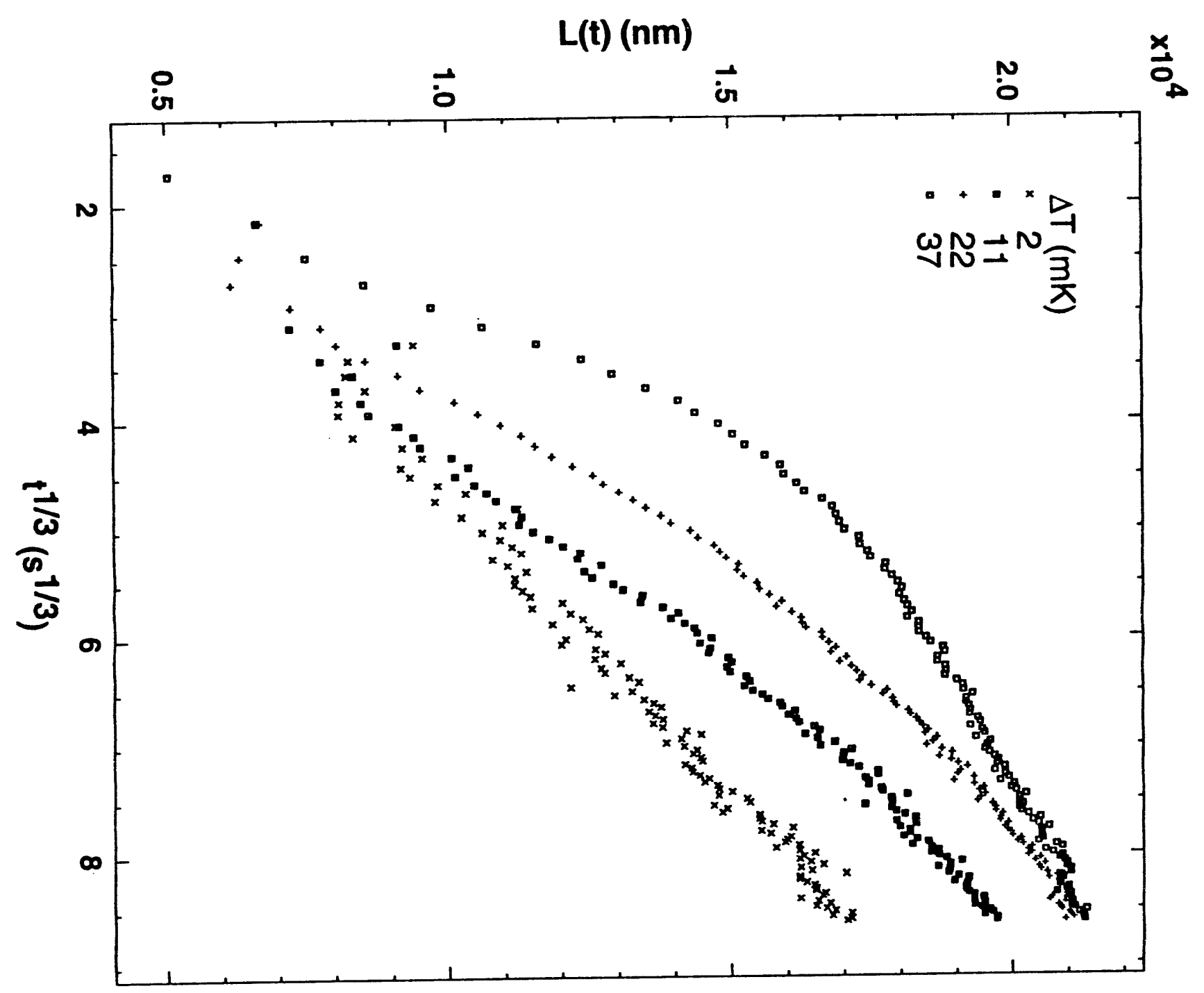




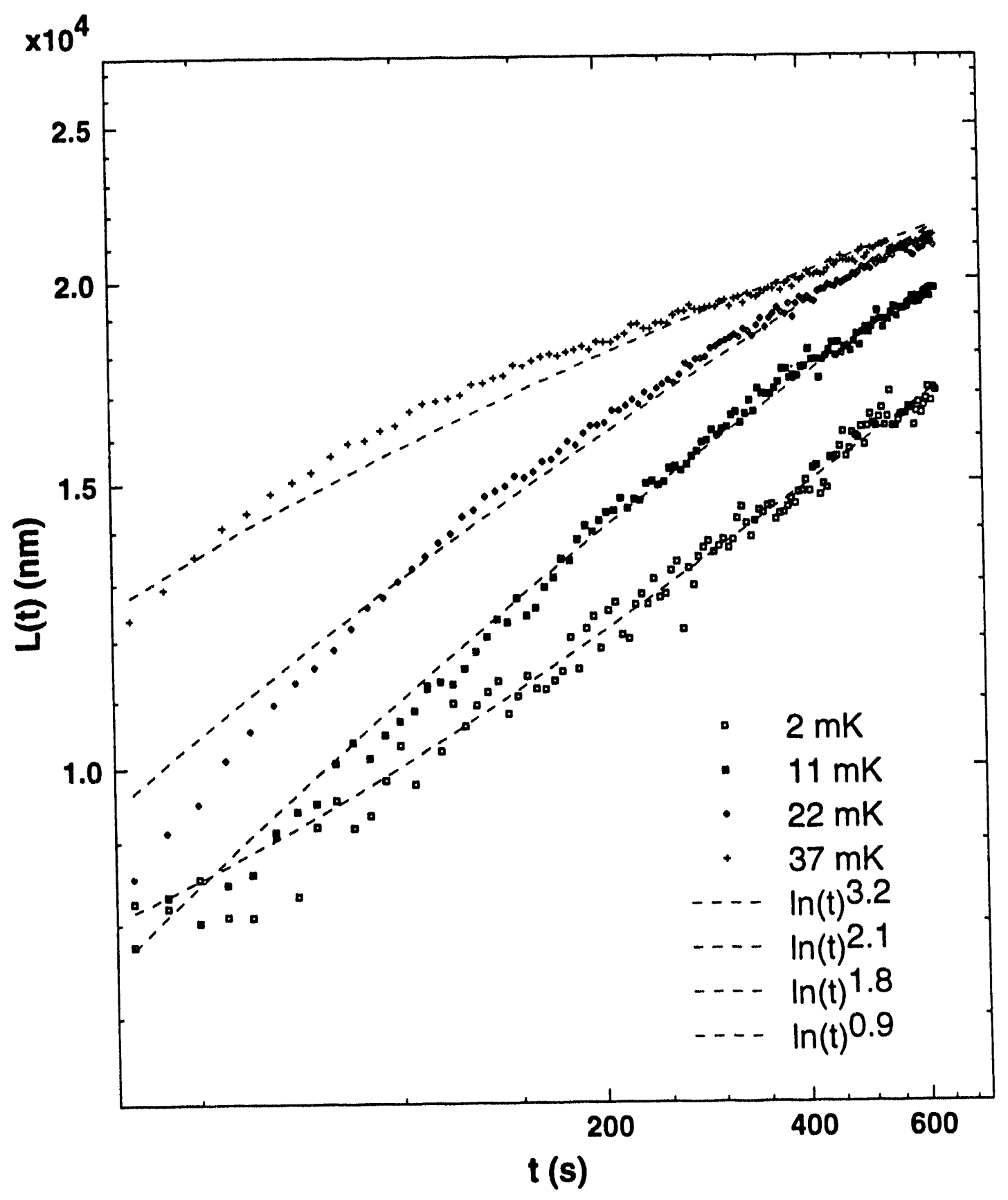




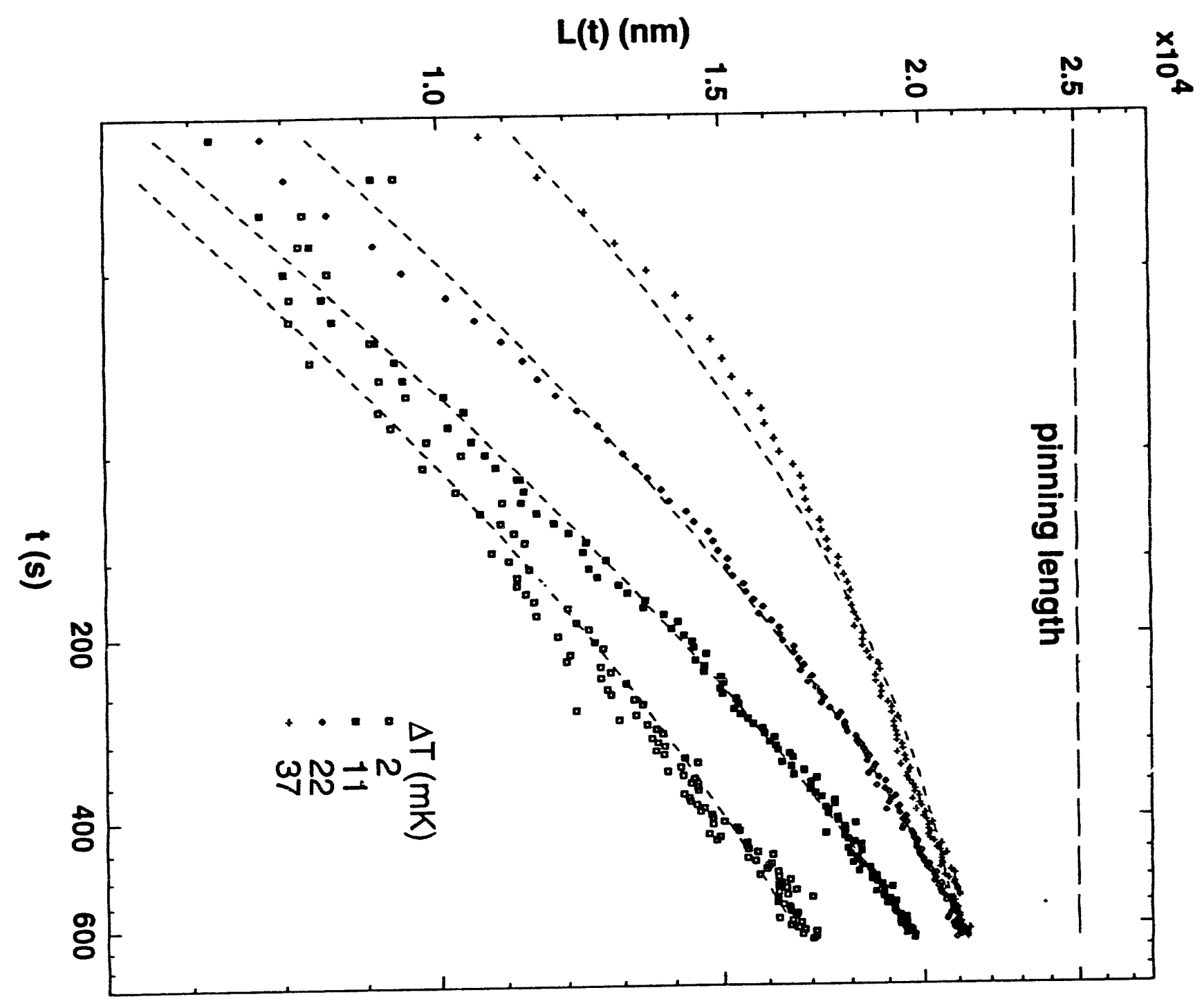




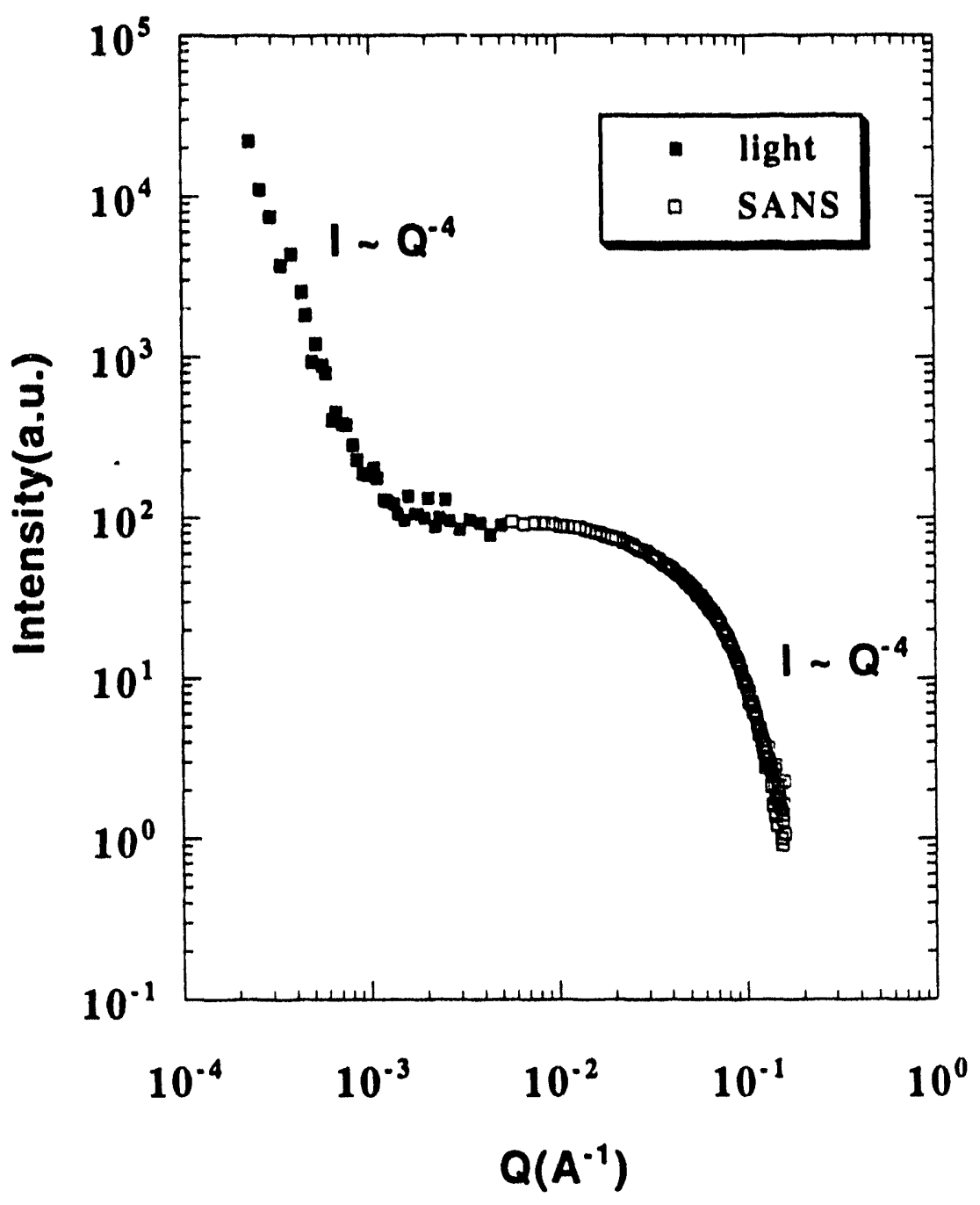



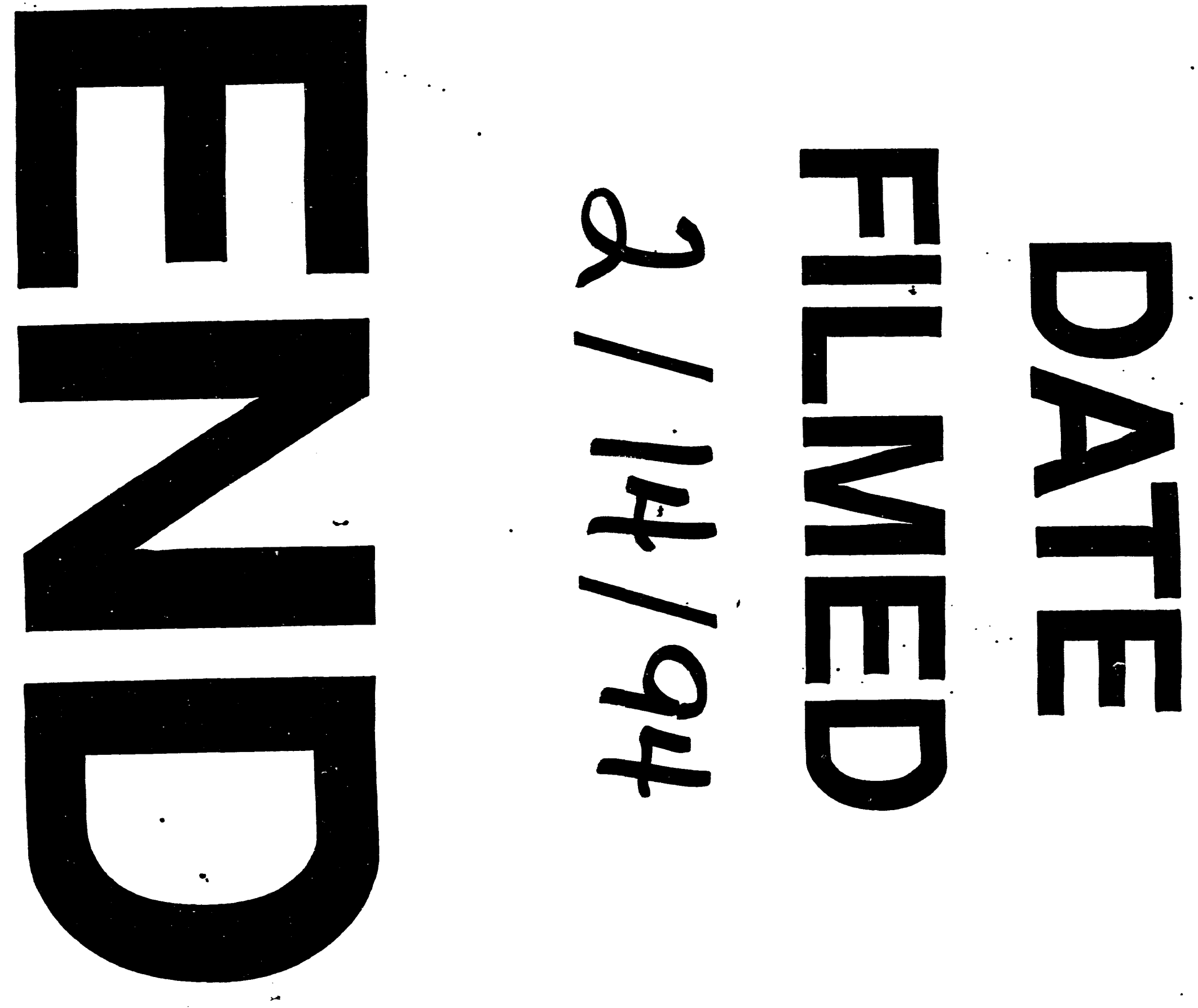
